\title{
Direct measurement of interatomic force gradients using an ultra-low-amplitude atomic force microscope
}

\author{
By Peter M. Hoffmann ${ }^{1}$, Ahmet Oral ${ }^{2}$, Ralph A. Grimble ${ }^{1}$, \\ H. ÖzG ̈̈r ÖzER ${ }^{2}$, Steve JefFerY ${ }^{1}$ And John B. PethicA ${ }^{1}$ \\ ${ }^{1}$ Department of Materials, University of Oxford, Parks Road, Oxford OX1 3PH, UK \\ ${ }^{2}$ Department of Physics, Bilkent University, Ankara, Turkey
}

Received 21 July 2000; accepted 23 November 2000

Interatomic force gradients between a $\mathrm{W}$ tip and a $7 \times 7$ reconstructed $\mathrm{Si}(111)$ surface were measured using an off-resonance, ultra-low-amplitude atomic force microscope (AFM) technique. The amplitudes used were less than $1 \AA$ (peak-to-peak), which allowed direct measurement of the interaction force gradients as a function of separation. The force gradient curves are shown to consist of an attractive van der Waals part and short-range attractive and repulsive interactions. The van der Waals background can be subtracted, leaving a short-range interaction with an energy parameter of $1.9-3.4 \mathrm{eV}$ and an interaction length-scale of $0.54-1.26 \AA$, characteristic of a single atomic bond. This correlates well with our observation of single-atom resolved force gradient images. In general, the interaction is reversible up to the zero intercept of the force gradient (inflection point of the energy). Beyond this point hysteresis tends to be observed and the onset of inelastic deformation can be clearly discerned. An analysis of the atomic scale contact gives reasonable values for the interfacial energy, yield strength, and the energy per atom needed to initiate plastic deformation.

Keywords: atomic force microscopy; interatomic potentials; nanomechanics; contact mechanics; atomic scale imaging

\section{Introduction}

Scanning probe microscopies are at the heart of nanoscience. The atomic force microscope (AFM) especially promises to be a key technique for measurement and manipulation of nanoscale volumes of matter down to the atomic scale. However, true atomic resolution in the AFM has only been achieved recently, so far by use of largeamplitude, frequency modulation techniques (Giessibl 1995; Kitamura \& Iwatsaku 1995; Ueyama et al. 1995). While providing high-resolution images, these techniques are limited as a quantitative spectroscopic tool, in atomic manipulation experiments and in measuring the nature of dissipative processes, which lie at the heart of much of nanotechnology. This is because the measured parameter (frequency shift $\Delta f$ ) is not related in a simple manner to either the interaction energy or the force. Mathematical deconvolution is needed in order to extract the force (Giessibl 1997; Gotsmann et al. 1999a; Dürig 1999; Hölscher et al. 1999; Ke et al. 1999), which relies on a number 
of assumptions such as the harmonic motion of the lever, a conservative interaction potential and general reversibility of the interaction. However, imaging with the large-amplitude AFM consistently involves a site-dependent damping (Molitor et al. 1999; Gotsmann et al. 1999b) which is associated with non-conservative interactions and hysteresis of the energy curve.

In this paper we report a non-contact atomic force microscopy technique that directly measures the force gradient of the tip-sample interaction using very small oscillation amplitudes of less than $1 \AA$ peak-to-peak (p-p) and sub-resonance oscillation frequencies. In this limit the measurement is linear and quasi-static. This enables us to measure directly complete force gradient curves up to the contact regime. In our technique the dynamic energy of the vibrating lever, and thus the maximum possible energy input into the tip-sample interaction region, is less than $0.1 \mathrm{eV}$ per cycle as opposed to $10-100 \mathrm{eV}$ in the case of large-amplitude, resonance-enhanced techniques (Anczykowski et al. 1999; Gotsmann et al. 1999b).

Since we measure the interaction directly, we can separate contributions due to van der Waals, electrostatic and short-range interactions without the need for any deconvolution in terms of frequency shifts (Guggisberg et al. 2000). In this way we can gain insight into the differences in interaction ranges that have been reported in the literature (Jarvis et al. 1996; Cross et al. 1998; Gotsmann et al. 1999b; Guggisberg et al. 2000) and the influence of tip geometry on high-resolution imaging. Moreover, we can quantify the effect of the relaxation of the tip and surface and also the behaviour in the contact regime, where we can clearly see the onset of plastic deformation and hysteresis in the force gradient curve. Inelastic deformation is observed when the inflection point of the interaction energy is surpassed. Therefore, in the non-contact region, the second derivative of the energy curve (the interaction stiffness) provides the most clear information, since it passes through its minimum and intercept before contact is established. In our technique, the interaction stiffness is measured directly. The determination of the interaction length-scale then only involves a suitable subtraction of the long-range interaction terms, while in largeamplitude methods the length-scale has to be inferred from taking the derivative of the deconvoluted frequency shift data after such a subtraction (Lantz et al. 2000).

\section{Experimental}

Our AFM technique relies on vibrating a stiff lever at sub-angstrom amplitudes, $A_{0}$, and sub-resonance frequencies and monitoring the changes in vibration amplitude of the lever which result from interactions. Since in this small amplitude limit the measurement is linear and quasi-static, a force balance readily shows that the interaction stiffness (negative of the force gradient) can be measured directly from the change in vibration amplitude (Jarvis et al. 1993) and is given by

$$
k_{\text {total }}=-\frac{\partial F_{\text {total }}}{\partial z}=\sum_{j} k_{\mathrm{i}}^{(j)}=k_{\text {lever }}\left(\frac{A_{0}}{A}-1\right),
$$

where $A_{0}$ is the amplitude in the absence of tip-surface interactions, $A$ is the measured amplitude, $k_{\text {total }}$ is the total measured tip-surface interaction stiffness, and $k_{\text {lever }}$ is the lever stiffness. The total interaction stiffness, $k_{\text {total }}$, is typically a sum of several contributions, $k_{\mathrm{i}}^{(j)}$. The linearity of the measurements was confirmed by the 
fact that higher harmonics of the driving frequency were extremely small even in the presence of rapidly varying force gradients, and by comparing with numerical models of the equation of motion (P. M. Hoffmann \& J. B. Pethica 2001, unpublished work).

To avoid snap to contact, high stiffness tungsten levers were used (typically about $100 \mathrm{~N} \mathrm{~m}^{-1}$ ). This necessitates the use of an ultra-high-sensitivity displacement sensor, which has been achieved by the use of a fibre interferometer with a sensitivity higher than $2 \times 10^{-4} \AA \sqrt{ } \mathrm{Hz}$. The details of the technique will be presented elsewhere (A. Oral et al. 2001, unpublished work). The lever stiffnesses were characterized by applying a known force via a calibrated test lever and monitoring the deflection of both the test and the measured lever by high-resolution laser interferometric techniques.

Atomic scale imaging was obtained using the (averaged) tunnelling current as the feedback parameter. The oscillation period of the lever was much faster than the time constant of the feedback loop, thus causing no problems while imaging. All measurements were performed in ultra-high vacuum (less than $10^{-10}$ mbar) on clean $7 \times 7$ reconstructed $\mathrm{Si}(111)$ surfaces. The $\mathrm{W}$ tips were cleaned by baking at $160{ }^{\circ} \mathrm{C}$ and field emitting at $\pm 10 \mathrm{~V}$ while the tip was fully retracted (by $c a .1 \mu \mathrm{m}$ ). Care was taken to move the tip a large lateral distance after field emitting and before commencing any imaging.

\section{Atomic and van der Waals force gradients}

Figure $1 a, b$ shows measurements of the interaction stiffness between a $\mathrm{W}$ tip and a clean $7 \times 7$ reconstructed $\mathrm{Si}(111)$ surface as a function of distance for two different tips (1 and 2 ) and simultaneously measured tunnelling currents (which will be discussed later). The free oscillation amplitudes were $0.42 \AA(\mathrm{p}-\mathrm{p})$ and $0.49 \AA(\mathrm{p}-\mathrm{p})$, respectively, and the amplitude did not exceed $0.7 \AA(\mathrm{p}-\mathrm{p})$ at any time during the measurement. The stiffnesses of the levers were 120 and $180 \mathrm{~N} \mathrm{~m}^{-1}$, respectively.

Now, the displacements of the piezo actuator are not identical to the local displacements of the tip. First of all, the bending of the cantilever structure has to be taken into account (Burnham 1993). Secondly, the relaxation of the tip and surface needs to be considered more carefully, since it depends not only on the magnitude of the force but also on its range. Longer-range forces act on a larger part of the tip and thus encounter a potentially larger stiffness. Van der Waals and electrostatic interactions are slowly varying on the scale of typical atomic bond distances and, thus, the correct stiffness to use is that of the macroscopic tip, which is quite large. The total deflection due to van der Waals forces can be calculated if we consider that the displacement is a function of the force acting on each layer of the tip, $\mathrm{d} F$, and the stiffness of the tip above each layer, $k_{\text {tip }}(h)$. For a van der Waals interaction acting on a tip we can write the total displacement in the following way (Israelachvili 1991):

$$
\delta L=\int_{0}^{\infty} \frac{\mathrm{d} F}{k_{\mathrm{tip}}(h)}=\int_{0}^{\infty} \frac{A_{\mathrm{H}} R^{2}(h)}{2(h+d)^{4}} \frac{\mathrm{d} h}{k_{\mathrm{tip}}(h)} .
$$

Here $A_{\mathrm{H}}$ is the Hamaker constant (taken to be $3 \times 10^{-19} \mathrm{~J}$ ), $h$ is the height along the tip, $d$ is the tip-surface distance, and $R(h)$ is the tip radius at tip height, $h$. The geometry used in equations (3.1)-(3.3) is illustrated in figure 2. The stiffness above

Proc. R. Soc. Lond. A (2001) 

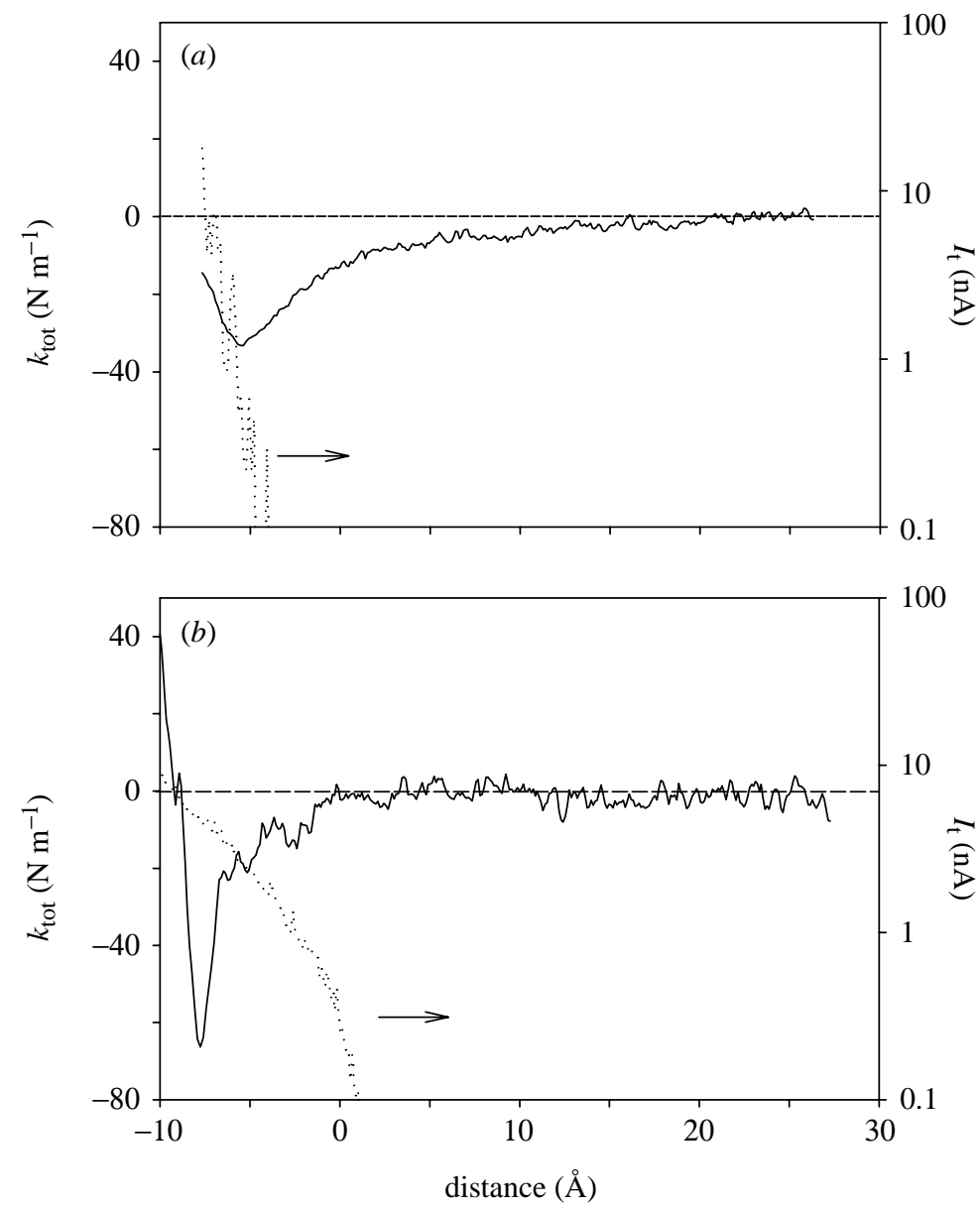

Figure 1. Measured interaction stiffness curves and simultaneously measured tunnelling currents versus distance for two different $\mathrm{W}$ levers and clean $\mathrm{Si}(111) 7 \times 7$ surfaces in ultra-high vacuum. Tip bias was $-2 \mathrm{~V}$ in both cases. The distance axis was corrected for the cantilever bending. (a) Measurement using cantilever/tip 1: the stiffness of the lever was $120 \mathrm{~N} \mathrm{~m}^{-1}$, the resonant frequency $\nu_{0}$ was $9.8 \mathrm{kHz}$, and the applied oscillation frequency $\nu_{\text {applied }}=4.7 \mathrm{kHz}$, free oscillation amplitude was $0.42 \AA$ (peak-peak). (b) Measurement using cantilever/tip 2: the stiffness of the lever was $180 \mathrm{~N} \mathrm{~m}^{-1}, \nu_{0}=16.2 \mathrm{kHz}, \nu_{\text {applied }}=4.7 \mathrm{kHz}$, and the free oscillation amplitude was $0.49 \AA$ (peak-peak). The total acquisition time was of the order of $10 \mathrm{~s}$.

each layer, $k_{\text {tip }}$, for a cone-shaped tip with cone angle $\alpha$, is given by

$$
k_{\text {tip }}(h)=c_{\text {tip }}^{-1}(h)=\left(\int_{h}^{\infty} \frac{\mathrm{d} h}{A(h) E}\right)^{-1}=\left(\int_{h}^{\infty} \frac{\mathrm{d} h}{E \pi h^{2} \tan ^{2} \alpha}\right)^{-1}=E \pi h \tan ^{2} \alpha,
$$

where $c_{\text {tip }}(h)$ is the tip compliance, $A(h)$ is the cross-sectional area at height $h$, and $E$ is the Young's modulus of the tip. Plugging (3.2) into (3.1) and integrating, we obtain, for the total tip displacement,

$$
\delta L=\frac{A_{\mathrm{H}}}{12 \pi E d^{2}} .
$$

Proc. R. Soc. Lond. A (2001) 


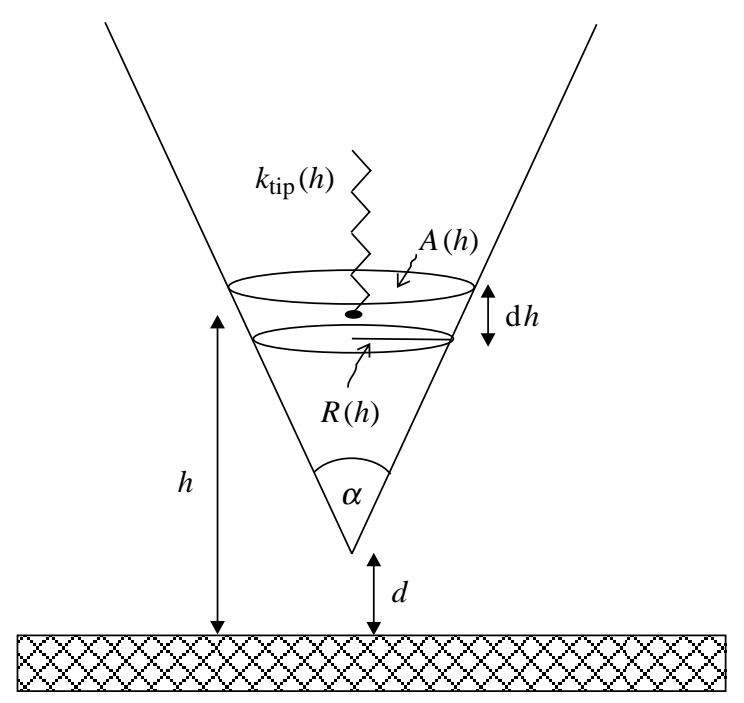

Figure 2. Geometry used in equations (3.1)-(3.3) to calculate the total tip relaxation due to long-range forces.

For a cone-shaped W tip we arrive at a displacement of $0.02 \AA$ at a typical equilibrium distance of $2 \AA$ and less for larger separations. The corresponding displacement of the surface can be estimated to be $c a .0 .04 \AA$, taking the lower modulus of silicon into account. This shows that the total surface-tip relaxation due to van der Waals interactions is unlikely to exceed $0.1 \AA$ and, consequently, will be neglected in the following analysis. The chosen geometry has little effect on these conclusions and similar results can be obtained for the similarly slowly varying electrostatic interactions which act on the surface of the conducting tip.

Long-range forces can be electrostatic and van der Waals. Electrostatic forces are expected to be dependent on the square of the bias voltage between tip and sample, $V_{\text {bias }}^{2}$ (Jeffery et al. 2000; Guggisberg et al. 2000). We analysed a variety of experimental force curves obtained at different voltages in the -0.25 to $-2 \mathrm{~V}$ range and found that the long-range interaction does not show any systematic dependence on $V_{\text {bias }}^{2}$ and is therefore mostly due to van der Waals interactions. This does not mean, however, that electrostatic forces are not present, only that they are much weaker or more slowly varying than the van der Waals forces.

Now, in order to separate out the van der Waals contribution it is necessary to determine the power-law dependence of the measured stiffness on the separation. A fourth-order power law (for the force gradient) corresponds to a truncated (circular) rod geometry for the macroscopic part of the tip, a third-order law indicates a spherical tip, and a cone-shaped truncated or spherically capped tip corresponds to mixed second- to fourth-order terms. In our case, we found that an exponent of 3.5-4 gave the best fit to the long-range data and we therefore chose a fourth-order power law. Real tip structures can be expected to be highly irregular and thus any attempt to model the background in such simple terms has to be an idealization. However, the shape of the modelled long-range interaction curve is essentially independent of the particular model, as both the distance offset and curvature of the curve are determined by the measured data. The van der Waals stiffness for a truncated, circular 
rod is given by

$$
k_{\mathrm{vdW}}=-\frac{A_{\mathrm{H}}}{2} \frac{R^{2}}{\left(d-d_{0}\right)^{4}},
$$

where $R$ is the radius at the (macroscopic) tip end and $d_{0}$ is a distance offset that allows for the fact that the absolute zero of the distance axis is unknown. Analysing the data shown in figure 1 we found $R=65.5 \pm 5.6 \mathrm{~nm}$ for tip 1 and $R=17.8 \pm 3.5 \mathrm{~nm}$ for tip 2. Averaged over several measured force curves obtained with the same two tips we found radii in the range of $52 \pm 13 \mathrm{~nm}$ and $32 \pm 18 \mathrm{~nm}$ for tips 1 and 2 , respectively. In the case of tip 1 the radius did not change much between measurements, while in the case of tip 2 we observed a slow but marked increase of the radius with the number of measurements (some of which included full contact and indentation of the tip). Figure $1 b$ shows a measurement that was obtained rather early in the 'life' of tip 2. It became 'blunter' later on. In general, the obtained values for $R$ were reproducible between measurements performed with the same tip. Thus, this seems to be a stable and reproducible process which gives mesoscopic geometric parameters that are physically realistic. The exact geometry is, of course, unknown.

Having subtracted the van der Waals background as described above, we are always left with a short-range attractive/repulsive interaction. We now need to take the relaxation of the outermost tip atoms into account (Pérez et al. 1998). This is done by rescaling the distance axis and the measured short-range stiffness according to (Burnham 1993, 1994):

$$
\begin{aligned}
& k_{\mathrm{sr}}=k_{\mathrm{m}}\left(1-\frac{k_{\mathrm{m}}}{k_{\mathrm{ts}}}\right)^{-1} \\
& z_{\mathrm{sr}}=z_{\mathrm{m}, \infty}+\int_{\infty}^{z_{\mathrm{m}}}\left(1+\frac{k_{\mathrm{sr}}}{k_{\mathrm{ts}}}\right)^{-1} \mathrm{~d} z
\end{aligned}
$$

where $z_{\mathrm{sr}}$ is relaxation-corrected distance (not the actual gap separation as there is always an arbitrary offset), $z_{\mathrm{m}}$ is the measured distance (after correcting for the lever movement), $k_{\mathrm{sr}}$ is the actual short-range interaction stiffness, $k_{\mathrm{m}}$ the measured short-range stiffness, and $k_{\mathrm{ts}}$ the combined tip/surface stiffness. Here we use stiffness values, $k_{\mathrm{ts}}$, obtained from the study of $\mathrm{Si}-\mathrm{Si}$ interactions by Pérez et al. (1998), but correcting for the fact that tungsten has a higher modulus. In particular, we took the stiffness of the Si surface to be $110 \mathrm{~N} \mathrm{~m}^{-1}$ and the stiffness of the $\mathrm{W}$ tip to be $300 \mathrm{~N} \mathrm{~m}^{-1}$. This gives a combined tip/surface stiffness, $k_{\mathrm{ts}}$, of $80.5 \mathrm{~N} \mathrm{~m}^{-1}$. It should be noted that values for the tip stiffness depend on the atomic configuration at the end of the tip, and, therefore, variations are possible. However, the values given by Pérez et al. (1998) are generally in agreement with other studies (Clarke et al. 1996).

The corrected short-range interactions are shown in figure 3 . To model the shortrange interaction we used a semi-empirical short-range interaction potential (Rose et al. 1984; Sutton 1993) given by

$$
k_{\text {int }}=\frac{\mathrm{d}^{2} U}{\mathrm{~d} z^{2}}=\frac{\left|E_{\mathrm{b}}\right|}{\lambda^{2}}\left(1-1.3 a+0.3 a^{2}-0.05 a^{3}\right) \mathrm{e}^{-a},
$$

where $E_{\mathrm{b}}$ is the minimum of the interaction potential (i.e. the bond energy), $\lambda$ is the characteristic length-scale (roughly the distance between the minimum of the 


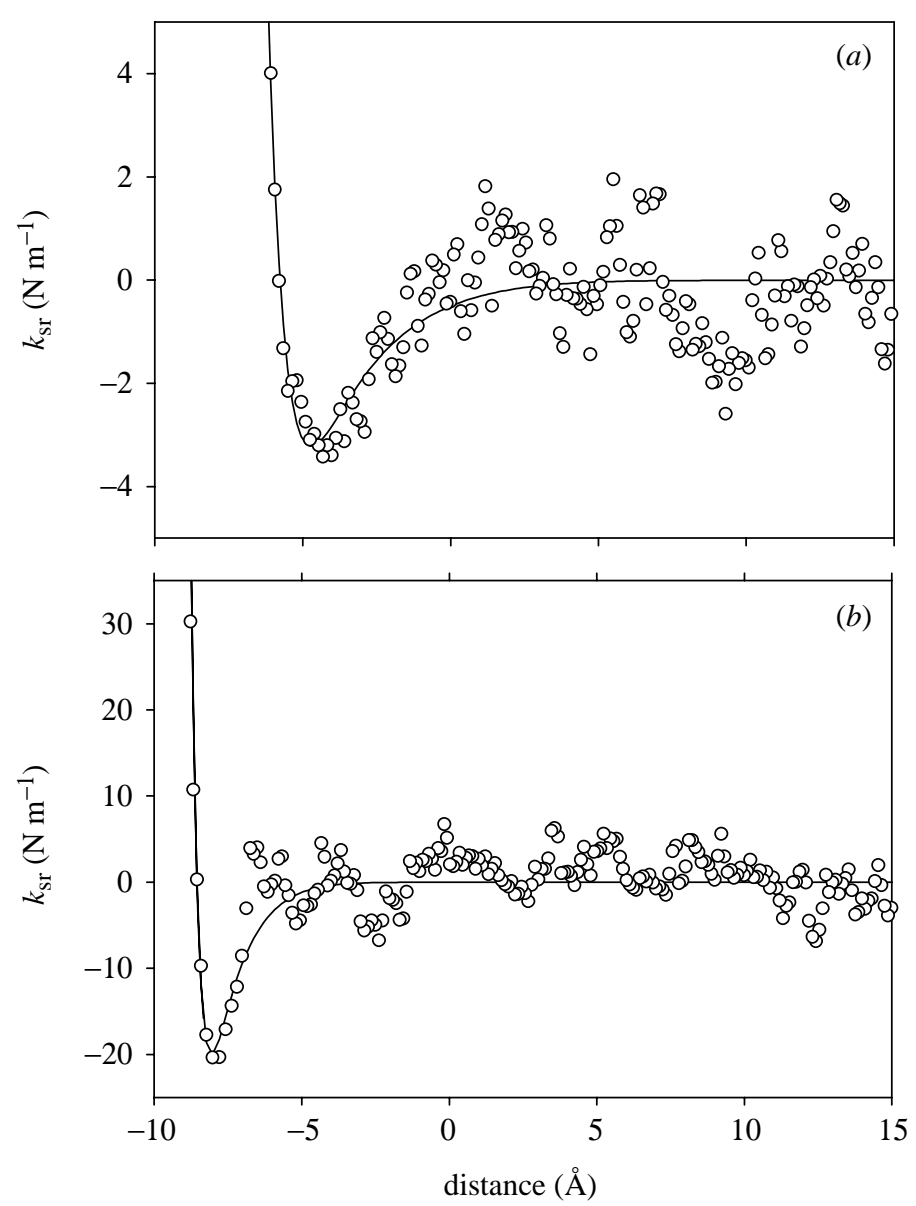

Figure 3. Interaction stiffness due to short-range covalent bonding interactions obtained by subtracting the long-range van der Waals background and correcting for the relaxation of tip and surface. (a) Tip 1: interaction energy $\left|E_{\mathrm{b}}\right|=1.9 \pm 1.0 \mathrm{eV}$, characteristic length-scale $\lambda=1.26 \pm 0.20 \AA$. (b) Tip 2: $\left|E_{\mathrm{b}}\right|=3.4 \pm 1.4 \mathrm{eV}, \lambda=0.54 \pm 0.08 \AA$.

interaction potential and its inflection point), and $a$ is the normalized distance given by

$$
a=\left(z-z_{0}\right) / \lambda
$$

where $z_{0}$ is a distance offset which results from the fact that the true zero of the distance axis is unknown. Fitting this potential to the short-range part of the force gradient (figure 3 ), the average length-scales were determined to be $1.14 \AA$ for tip 1 and $0.53 \AA$ for tip 2 . The energy parameter, $\left|E_{\mathrm{b}}\right|$, was found to be 2.41 or $3.18 \mathrm{eV}$, respectively. Averaged over several measurements performed with the same tips we found $\lambda=1.26 \pm 0.20 \AA,\left|E_{\mathrm{b}}\right|=1.9 \pm 1.0 \mathrm{eV}$ for tip 1 and $\lambda=0.54 \pm 0.08 \AA$, $\left|E_{\mathrm{b}}\right|=3.4 \pm 1.4 \mathrm{eV}$ for tip 2 . The measured values for the interaction range and energy compare well with theoretical values (Pérez et al. 1998). Although these measurements were done at room temperature, a thermally induced broadening of the interaction curve, as recently suggested, was not observed (Lantz et al. 2000). We conclude that we are directly measuring single bonds' mechanical properties. The 
Table 1. Parameters obtained from measurements using tips 1 and 2

\begin{tabular}{lcc}
\hline & cantilever/tip 1 & cantilever/tip 2 \\
\hline lever stiffness $\left(\mathrm{N} \mathrm{m}^{-1}\right)$ & 120 & 180 \\
resonance frequency $\nu_{0}(\mathrm{kHz})$ & 9.8 & 16.2 \\
applied frequency $\nu_{\text {applied }}(\mathrm{kHz})$ & 4.7 & 4.7 \\
free amplitude $(\AA)(\mathrm{p}-\mathrm{p})$ & 0.42 & 0.49 \\
van der Waals radius from figure $1(\mathrm{~nm})$ & $65.5 \pm 5.6$ & $17.8 \pm 3.5$ \\
average van der Waals radius $(\mathrm{nm})$ & $52 \pm 13$ & $32 \pm 18$ \\
short-range energy parameter $\left|E_{\mathrm{b}}\right|(\mathrm{eV})$ & $1.9 \pm 1.0$ & $3.4 \pm 1.4$ \\
short-range length-scale $\lambda(\AA)$ & $1.26 \pm 0.20$ & $0.54 \pm 0.08$ \\
\hline
\end{tabular}

measured parameters for tips 1 and 2 are summarized in table 1 . We now consider the relation of these observations to atomic scale imaging.

\section{Imaging and the relative contribution of short-range interactions}

The attractive length range and the relative strength of the van der Waals interaction greatly influence the lateral resolution while imaging. An indicator for the attractive length range of the interaction is the position of the onset of the simultaneously measured tunnelling current. As seen in figure $1 a$, the current onset in the case of tip 1 (large $R$, 'blunt') only occurred after the onset of the attractive force gradient. Now, a key observation is that only in cases where we observed the onset of the tunnelling current before a significant attractive force gradient (tip 2) were we able to achieve atomic resolution imaging. The relatively large difference between the onset of the tunnelling current in figure $1 a, b$ is quite surprising. At this point, we can only speculate about its origin, but it should be noted that not only the onset but also the apparent barrier height (slope of the tunnelling current) is quite different in the two figures. A possible explanation might involve the detailed chemical nature of the tip, i.e. the type of atom interacting with the surface.

Figure 4 shows topographic scanning tunnelling microscopy (STM) and simultaneously acquired force gradient images of $\operatorname{Si}(111) 7 \times 7$. Averaged over several images obtained under identical conditions, the average topographical (STM) and force gradient corrugations between adatom and cornerhole for tip 2 were $1.07 \pm 0.18 \AA$ and $8.4 \pm 3.1 \mathrm{~N} \mathrm{~m}^{-1}$, respectively, while in the case of tip 1 no atomic resolution could be achieved. An important aspect in imaging is evidently the need for a relatively strong short-range part of the total interaction to give significant contrast at atomic resolution. In the case of tip 1 only a very small fraction of the total force gradient (less than 10\%) was due to short-range interactions, and, therefore, the force gradient imaging resolution can be expected to be seriously diminished.

The geometry of the tip and, thus, the relative contribution of van der Waals interactions and short-range interactions greatly influences the overall apparent interaction length-scales (Buldum et al. 1999), the energies of the total measured force gradient, and the contrast in atomic scale imaging. It is clearly an essential factor in explaining the wide variability seen in the earlier literature. Using the measured parameters for tips 1 and $2(R=17.8$ and $65.5 \mathrm{~nm})$, we investigated this influence by calculating theoretical interaction stiffness curves composed of a short-range 

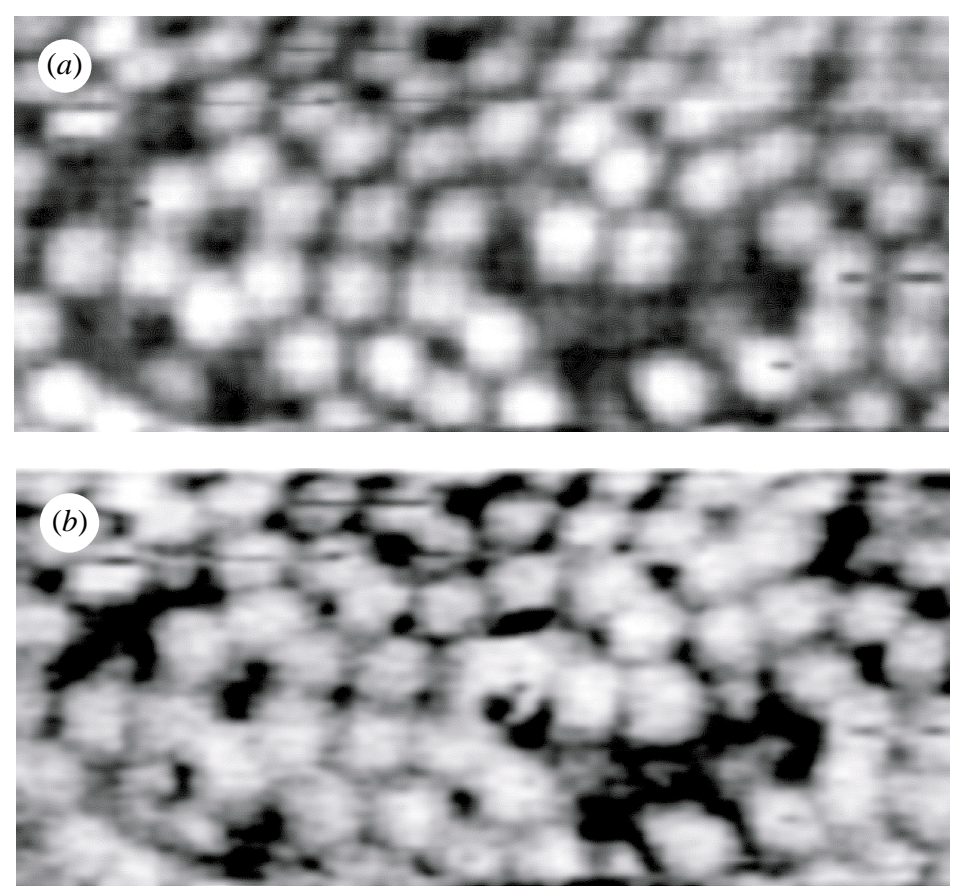

Figure 4. Simultaneously acquired STM topography $(a)$ and force gradient image $(b)$ of $\mathrm{Si}(111) 7 \times 7$ using a free amplitude of $0.4 \AA$ (peak-peak). Image size is $70 \times 32 \AA^{2}$, acquisition time was $10 \mathrm{~min}$.

covalent interaction (equation (3.6)) and a van der Waals interaction (given by equation (3.4)). In figure 5, the ratio between the short-range stiffness minimum and the total stiffness minimum (given as a percentage) is plotted versus the relative strength of the van der Waals interaction at the tip end, $s$. The relative strength is given by $s=R^{2} / \Delta d^{4}$, where $\Delta d=d_{0}-z_{0}$ is a measure for the distance between the mesoscopic (van der Waals) tip and the actual (covalent) tip end. The deflection of the lever and the relaxation of tip and surface were also taken into account. At large offsets or small radii (small $s$ ), the short-range interaction dominates the stiffness minimum. As the offset decreases or the radius increases (and, thus, $s$ increases), the van der Waals contribution becomes more dominant. The relative strength of the short-range interaction depends on its length range and energy. In the case of tip 2 , the short-range interaction continues to dominate the total stiffness much longer than in the case of tip 1 due to its much shorter length range and thus larger maximum force gradient. This is expected to have consequences for the contrast obtained when using these tips in imaging.

The inset to figure 5 shows the influence of the relative strength, $s$, on the apparent attractive length-scale of the total force gradient. The attractive range, $l_{\mathrm{attr}}$, is given here by the distance away from the surface over which the force gradient has decayed to $1 / \mathrm{e}$ of the maximum. For a purely short-range interaction (equation (3.6)) with a repulsive range of $\lambda=1.26 \AA$ acting on lever/tip 1 we would expect an apparent attractive range of $3.00 \AA$ and for lever/tip 2 with $\lambda=0.54 \AA$ we would expect $l_{\text {attr }}=0.85 \AA$. It is interesting to note that for very small offsets (and thus strong influence of the van der Waals forces, large $s$ ), the apparent length range begins to 


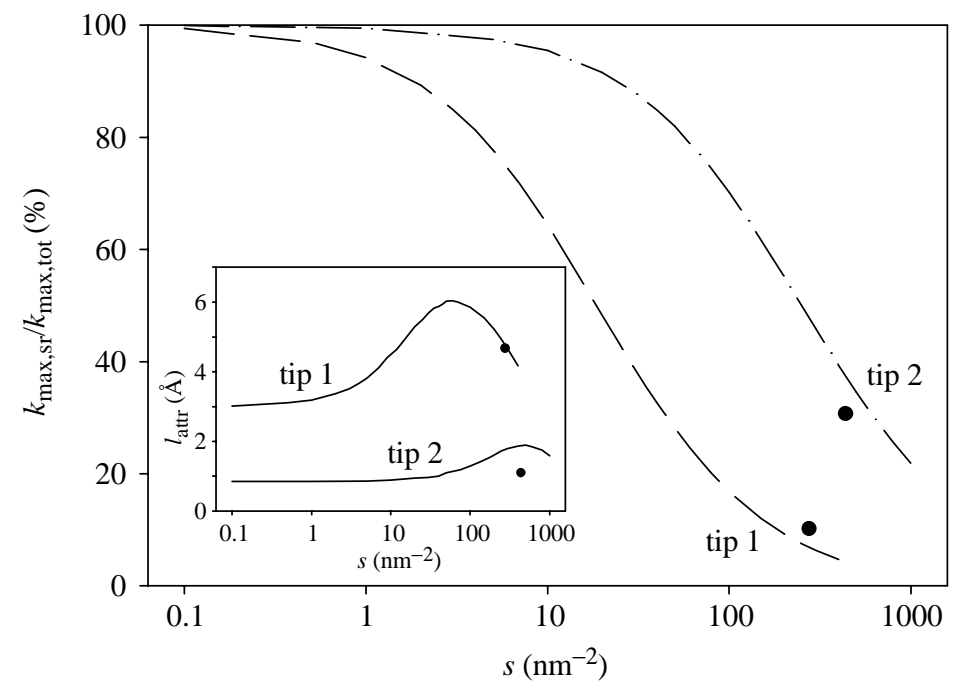

Figure 5. Proportion of the total measured interaction stiffness that is due to short-range interactions plotted versus the strength of van der Waals interaction, $s$ (see text for an explanation). Lower curve $(\operatorname{tip} 1): R=65.5 \mathrm{~nm}, \lambda=1.26 \AA,\left|E_{\mathrm{b}}\right|=1.90 \mathrm{eV}$. Upper curve $(\operatorname{tip} 2): R=17.8 \mathrm{~nm}$, $\lambda=0.54 \AA,\left|E_{\mathrm{b}}\right|=3.42 \mathrm{eV}$. Dots indicate measurements taken from figures 1 and 3 . The inset shows the apparent attractive length range $l_{\text {attr }}$ versus $s$ for the same parameters $R, \lambda$ and $\left|E_{\mathrm{b}}\right|$.

shorten again. This is due to the high curvature of the van der Waals interaction stiffness when the offset is quite low. However, we should note that at very small separations between the tip and surface, the van der Waals force may be reduced and the usual power law will not apply (Heinrichs 1973, 1975; Buhl 1976; Hartmann 1990), so the observation of a very short interaction range can generally be attributed to covalent interactions.

\section{Atomic scale contact and inelasticity}

Using our AFM technique it is also possible to continue the tip-surface approach until repulsion terms become significant. We can thus measure and analyse contact stiffnesses. Figure 6 shows forward (moving toward the surface) and reverse (moving away from the surface) scans using tip 2 which were measured up to the contact regime. These scans were obtained in the early stages of the tip's use, when it was still rather sharp. The forward and reverse scans are significantly different and greatly displaced with respect to each other. In general, we observed hysteresis between forward and reverse scans when the zero intercept of the force gradient (inflection point of the energy or minimum of the force) was exceeded. Once this point is passed, inelastic processes are taking place. In contrast, if the measurement is reversed before the inflection point of the energy the forward and reverse scans coincide except for a very small displacement due to drift. These observations put a severe limitation on large-amplitude, frequency-shift-based AFM methods, since their interpretation relies on a single-valued and reversible interaction curve. Thus, meaningful results can only be obtained with these techniques if the minimum of the force curve is not passed at any time during the motion of the cantilever. 


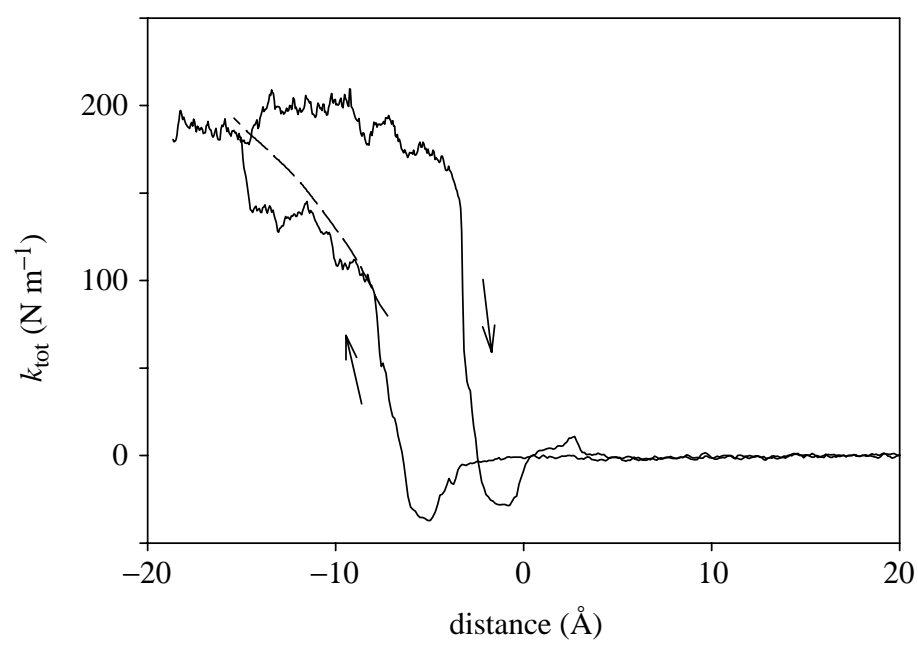

Figure 6. Forward and reverse scans measuring the interaction/contact stiffness between a $\mathrm{W}$ tip and a $\mathrm{Si}(111) 7 \times 7$ surface in UHV. There is large hysteresis between the two scans due to non-conservative processes in the contact region. The dashed line shows a fit according to equations (5.1)-(5.4) with $E^{\star}=105 \mathrm{GPa}, F_{\mathrm{c}}=24 \mathrm{nN}, R=1 \mathrm{~nm}$ and $\gamma=1.9 \mathrm{~J} \mathrm{~m}^{-2}$.

Even closer to the surface, the force gradient curve starts to bend over and the curve is interrupted by distinct jumps, i.e. sudden changes in stiffness without a large change in the tip-surface separation. In this region the positive (repulsive) stiffness exceeds the intrinsic stiffness of the tip and the overall stiffness is no longer dominated by interfacial interactions but by deformation of the bulk. This latter region can be analysed by continuum contact stiffness models, and, in the context of this section of the paper, we define this region as the contact region. Since we measure the interaction directly, we can analyse the contact stiffness to provide a quantitative picture of the contact mechanics of a sharp tip. As seen in figure 6, there is a slow increase in the stiffness once contact has been established interrupted by sudden upward jumps. These jumps could be associated with atomic rearrangement in the tip region resulting in a more stable (higher intrinsic stiffness) tip configuration. The slow increase before each jump can be analysed using the Johnson-KendallRoberts/Derjaguin-Muller-Toporov (JKR/DMT) (Johnson et al. 1971; Derjaguin et al. 1975; Johnson 1985) model of contact with adhesion. In this model there are two unknowns, the tip radius, $R$, and the interfacial energy, $\gamma$, which are related to the pull-off force via (Derjaguin et al. 1975; Israelachvili et al. 1980; Israelachvili 1991)

$$
F_{\mathrm{c}}=4 \pi \gamma R \text {. }
$$

The contact radius, $r_{\mathrm{c}}$, can be calculated from (Johnson 1985)

$$
\left(F-\frac{4 E^{\star} r_{\mathrm{c}}^{3}}{3 R}\right)^{2}=16 \pi \gamma E^{\star} r_{\mathrm{c}}^{3}
$$

where $E^{\star}$ is given by

$$
\frac{1}{E^{\star}}=\frac{1-\nu_{\mathrm{Si}}^{2}}{E_{\mathrm{Si}}}+\frac{1-\nu_{\mathrm{W}}^{2}}{E_{\mathrm{W}}} .
$$

Proc. R. Soc. Lond. A (2001) 
Here, $E_{\mathrm{Si}}, E_{\mathrm{W}}$ are the Young's moduli, and $\nu_{\mathrm{Si}}, \nu_{\mathrm{W}}$ are the Poisson ratios of silicon and tungsten, respectively. The contact stiffness, $k_{\mathrm{c}}$, is given by

$$
k_{\mathrm{c}}=2 E^{\star} r_{\mathrm{c}}-\frac{4\left(\gamma E^{\star} \pi\right)^{1 / 2} r_{\mathrm{c}}}{(2 / R) r_{\mathrm{c}}^{3 / 2}-\left(\pi \gamma / E^{\star}\right)^{1 / 2}} .
$$

The first term on the right-hand side of equation (5.4) is the Hertzian contact stiffness, whereas the second term represents a correction due to adhesion.

In order to analyse the experimental data, we first obtained a value for $F_{\mathrm{c}}$ from the tip retraction scan by measuring the size of the jump of the cantilever as it 'snapped' off the surface. In this case we measured $F_{\mathrm{c}}=24 \mathrm{nN}$. This determined the product $\gamma R$ (equation (5.1)). The force, $F$, was calculated as a function of distance by integrating the force gradient curve. Then, starting with a trial value for either $\gamma$ or $R$, equation (5.2) provided a value for $r_{\mathrm{c}}$ for every point along the curve, which was used in equation (5.4) to calculate $k_{\mathrm{c}}$ as a function of distance. Thus all parameters are determined from the measured data and there is only one adjustable parameter (either $\gamma$ or $R$ ). The resulting fit shown in figure 6 is very sensitive to the choice of $\gamma$ (and thus $R$ ): even a $10 \%$ change in $\gamma$ leads to a visibly worse fit of the experimental data. The best fit (the dashed line in figure 6) gives $\gamma=1.9 \mathrm{~J} \mathrm{~m}^{-2}$ and $R=1 \mathrm{~nm}$. Integrating over the stiffness, the elastic energy stored in the contact can be calculated. The initial number of atoms in contact with the surface can be estimated from the contact radius to be $c a .25$, which increases to $c a .65$ at the reversal point. It is found that the elastic energy per atom increases to $c a .0 .2-0.6 \mathrm{eV}$ per tip atom before a jump in the stiffness occurs. The contact pressure at the first jump is of the order of 7-10 GPa, which is comparable with the yield strength of silicon or tungsten. This model yields very reasonable values, despite the fact that the contact radius is of the same order as the tip radius $(c a .1 \mathrm{~nm})$ and the number of atoms in the contact is very small.

\section{Conclusions}

We have demonstrated that an off-resonance, sub-angstrom amplitude AFM technique can be used to measure directly short- and long-range interactions including hard contact. In addition, true atomic resolution can be achieved with this technique in force gradient imaging. The separation of different contributions to the total interaction stiffness is straightforward as no deconvolution of frequency shifts is needed. In addition, the energy input into the tip/surface region is minimal and non-conservative processes can be measured easily. This opens up a large number of applications in tribology, plasticity and atomic manipulation that are not accessible with large-amplitude methods. Analysing the data that we obtained, reasonable values for a number of physical parameters were obtained. These include the energy parameter and length range of covalent short-range interactions between $\mathrm{W}$ and $\mathrm{Si}$, the interfacial energy between $\mathrm{W}$ and $\mathrm{Si}$, and the elastic energies and pressures needed to initiate plastic deformation in small volumes of atoms. All of these measured values compare well with theoretical or known values.

\section{References}

Anczykowski, B., Gotsmann, B., Fuchs, H., Cleveland, J. P. \& Elings, V. B. 1999 How to measure energy dissipation in dynamic mode atomic force microscopy. Appl. Surf. Sci. 140, 376-382.

Proc. R. Soc. Lond. A (2001) 
Buhl, W. 1976 On the theory of van der Waals interactions. Z. Phys. B 23, 221-232.

Buldum, A., Ciraci, S., Fong, C. Y. \& Nelson, J. S. 1999 Interpretation of long-range interatomic force. Phys. Rev. B 59, 5120-5125.

Burnham, N. A. 1993 Apparent and true feature heights in force microscopy. Appl. Phys. Lett. 63, 114-116.

Burnham, N. A. 1994 Accounting for the stiffnesses of the probe and sample in scanning probe microscopy. J. Vac. Sci. Technol. B 12, 2219-2221.

Clarke, A. R. H., Pethica, J. B., Nieminen, J. A., Besenbacher, F., Lægsgaard, E. \& Stensgaard, I. 1996 Quantitative scanning tunnelling microscopy at atomic resolution: influence of forces and tip configuration. Phys. Rev. Lett. 76, 1276-1279.

Cross, G., Schirmeisen, A., Stalder, A., Grütter, P., Tschudy, M. \& Dürig, U. 1998 Adhesion interaction between atomically defined tip and sample. Phys. Rev. Lett. 80, 4685-4688.

Derjaguin, B. V., Muller, V. M. \& Toporov, Y. P. 1975 Effect of contact deformations on the adhesion of particles. J. Colloid Interface Sci. 53, 314-326.

Dürig, U. 1999 Relations between interaction force and frequency shift in large-amplitude dynamic force microscopy. Appl. Phys. Lett. 75, 433-435.

Giessibl, F. J. 1995 Atomic resolution of the silicon(111)- $(7 \times 7)$ surface by atomic force microscopy. Science 267, 68-71.

Giessibl, F. J. 1997 Forces and frequency shifts in atomic-resolution dynamic-force microscopy. Phys. Rev. B 56, 16 010-16 015.

Gotsmann, B., Anczykowski, B., Seidel, C. \& Fuchs, H. 1999a Determination of tip-sample interaction forces from measured dynamic force spectroscopy curves. Appl. Surf. Sci. 140, 314-319.

Gotsmann, B., Seidel, C., Anczykowski, B. \& Fuchs, H. 19996 Conservative and dissipative tip-sample interaction forces probed with dynamic AFM. Phys. Rev. B 60, 11 051-11 061.

Guggisberg, M., Bammerlin, M., Loppacher, Ch., Pfeiffer, O., Abdurixit, A., Barwich, V., Bennewitz, R., Baratoff, A., Mayer, E. \& Güntherodt, H.-J. 2000 Separation of interactions by noncontact force microscopy. Phys. Rev. B 61, 11 151-11 155.

Hartmann, U. 1990 Manifestation of zero-point quantum fluctuations in atomic force microscopy. Phys. Rev. B 42, 1541-1546.

Heinrichs, J. 1973 Non-local effects in the macroscopic theory of van der Waals and adhesive forces. Solid State Commun. 13, 1595-1598.

Heinrichs, J. 1975 Theory of van der Waals interactions between metal surfaces. Phys. Rev. B 11, 3625-3636.

Hölscher, H., Allers, W., Schwarz, U. D., Schwarz, A. \& Wiesendanger, R. 1999 Determination of tip-sample interaction potentials by dynamic force spectroscopy. Phys. Rev. Lett. 83, 47804783.

Israelachvili, J. N. 1991 Intermolecular and surface forces. Academic.

Israelachvili, J. N., Perez, E. \& Tandon, R. K. 1980 On the adhesion force between deformable solids. J. Colloid Interface Sci. 78, 260-261.

Jarvis, S. P., Oral, A., Weihs, T. P. \& Pethica, J. B. 1993 A novel force microscope and point contact probe. Rev. Sci. Instrum. 64, 3515-3520.

Jarvis, S. P., Yamada, H., Yamamoto, S.-I., Tokumoto, H. \& Pethica, J. B. 1996 Direct mechanical measurement of interatomic potentials. Nature 384, 247-249.

Jeffery, S., Oral, A. \& Pethica, J. B. 2000 Quantitative electrostatic force measurement in AFM. Appl. Surf. Sci. 157, 280-284.

Johnson, K. L. 1985 Contact mechanics. Cambridge University Press.

Johnson, K. L., Kendall, K. \& Roberts, A. D. 1971 Surface energy and the contact of elastic solids. Proc. R. Soc. Lond. A 324, 301-313.

Ke, S. H., Uda, T. \& Terakura, K. 1999 Quantity measured in frequency-shift-mode atomic-force microscopy: an analysis with a numerical model. Phys. Rev. B 59, 13 267-13272.

Proc. R. Soc. Lond. A (2001) 
Kitamura, S. \& Iwatsaku, M. 1995 Observation of $7 \times 7$ reconstructed structure on the silicon (111) surface using ultrahigh vacuum noncontact atomic force microscopy. Jap. J. Appl. Phys. 34, L145-L148.

Lantz, M. A., Hug, H. J., van Schendel, P. J. A., Hoffmann, R., Martin, S., Baratoff, A., Abdurixit, A., Güntherodt, H.-J. \& Gerber, Ch. 2000 Low temperature scanning force microscopy of the $\mathrm{Si}(111)-(7 \times 7)$ surface. Phys. Rev. Lett. 84, 2642-2645.

Molitor, S., Güthner, P. \& Berghaus, T. 1999 Contrast inversion in dynamic force microscopy on silicon(111) $7 \times 7$ and gold(111) $23 \times \sqrt{3}$. Appl. Surf. Sci. 140, 276-280.

Pérez, R., S̆tich, I., Payne, M. C. \& Terakura, K. 1998 Surface-tip interactions in noncontact atomic-force microscopy on reactive surfaces: Si(111). Phys. Rev. B 58, 10 835-10849.

Rose, J. H., Smith, J. R., Guinea, F. \& Ferrante, J. 1984 Universal features of the equation of state of metals. Phys. Rev. B 29, 2963-2969.

Sutton, A. P. 1993 Electronic structure of materials. Oxford: Clarendon.

Ueyama, H., Ohta, M., Sugawara, Y. \& Morita, S. 1995 Atomically resolved InP(110) surface observed with noncontact ultrahigh vacuum atomic force microscope. Jap. J. Appl. Phys. 4, L1086-L1088. 